\title{
¿Influyen las opciones político-religiosas en las actitudes hacia las personas mayores?
}

\section{Do political-religious choices influence attitudes towards older people?}

\author{
Eliezer Pazos, José Ma Faílde, Laura Ruiz, María D. Dapía, Miguel A. Vázquez \\ Universidade de Vigo.
}

\begin{abstract}
Resumen
El objetivo de este trabajo ha sido evaluar la relación que existe entre la práctica religiosa y la identidad política con los conocimientos y actitudes viejistas. La muestra estuvo integrada por 445 participantes distribuidos en tres grupos: jóvenes (18-35 años), adultos (36-64) y adultos mayores $(>65)$. Los instrumentos utilizados para llevar a cabo el estudio fueron la Subescala "Actitudes hacia el propio envejecimiento" y los cuestionarios: "The Facts on Aging Quiz-1", Características Negativas de la vejez y otro de datos sociodemográficos. Los resultados confirman que las dos variables analizadas presentan importancia en la modulación de los estereotipos hacia el envejecimiento.

Palabras clave: conocimientos, estereotipos, religiosidad, identidad política, viejismo.
\end{abstract}

\begin{abstract}
The objective of this work has been to evaluate the relationship between religious practice and political identity with knowledge and attitudes about old people. The sample is formed by 445 participants divided into three groups: young (18-35 years), adults (36-64) and older adults $(>65)$. The instruments used to carry out the study were the subscale "Attitudes Towards Aging" and the questionnaires: "The Facts on Aging Quiz-1", Negative Characteristics of Old Age and other about Sociodemographic Data. The results confirm that the two variables analyzed are important in the modulation of stereotypes towards aging.

Keywords: knowledge, stereotypes, religiosity, political identity, ageism.
\end{abstract}

Son diversos los estudios que demuestran que la población presenta un nivel de conocimientos deficientes sobre el envejecimiento y sus procesos asociados (Palmore, 1992; Villar y Triadó, 2000).

Para hablar sobre las actitudes hacia el envejecimiento, es necesario conocer el significado de las mismas. Así, las actitudes, tal y como las definen Eaagly y Chaiken (2005), son tendencias psicológicas que se expresan evaluando un ente determinado con cierto grado de favorabilidad o desfavorabilidad. Además, según Gaviria, López y Cuadrado (2013), éstas se estructuran en tres componentes diferenciados: 1) cognitivo, que hace referencia a los conocimientos; 2) afectivo, a las emociones y 3) conductual, al comportamiento. Existen por tanto: actitudes basadas en información cognitiva, que es donde situaríamos los estereotipos; actitudes basadas en información afectiva; actitudes basadas en información conductual, donde se incluyen las actitudes discriminatorias $\mathrm{y}$, por último, influencias biológicas y del contexto. Sin embargo, todas ellas son susceptibles de ser cambiadas o modificadas porque las actitudes son adquiridas, es decir, se aprenden.

El viejismo, al igual que los otros dos grandes -ismos, que son el sexismo y el racismo, es un tipo de discriminación que consiste en atribuir estereotipos sistemáticos y discriminatorios contra las personas, en este caso, por el simple hecho de ser mayores. Éstos se reflejan en conductas como el desdén, el desagrado, el insulto, o simplemente evitando la cercanía o el contacto físico. Además, estas conductas aprendidas llevan interiorizándose tanto tiempo sin cuestionar su validez, que hasta las propias personas mayores terminan atribuyéndose elementos negativos a sí mismas, es decir, autodiscriminándose.

La evidencia empírica demuestra que los programas intergeneracionales, la acción social, la formación de profesionales, y la educación de las personas mayores y sus familias a este respecto, contribuyen a reducir las actitudes viejistas.

El objetivo del presente estudio ha sido conocer en qué medida influye la práctica religiosa y la opción política en los estereotipos que se tienen hacia las personas mayores en tres grupos de edad diferenciados: jóvenes, personas adultas y personas mayores.

\section{Método}

En el presente apartado, referimos cuál ha sido el método empleado para llevar a cabo la investigación. Para ello, definimos la muestra, instrumentos y procedimiento utilizado en el estudio.

\section{Participantes}

Participaron un total de 445 personas gallegas mayores de 18 años que se distribuyeron en tres grupos de edad diferenciados: 145 jóvenes de entre 18 y 35 años (32,60\%), 154 personas adultas de entre 36 y 64 $(34,60 \%)$, y 146 personas mayores de 65 años $(32,80 \%)$. 


\section{Instrumentos}

Los instrumentos utilizados para llevar a cabo la investigación fueron:

Escala Geriátrica Multidimensional de Filadelfia (Lawton, 1975). Es la escala recomendada por grupos de expertos de la British Geriatrics Society y el American Nacional Institute of Aging para la medición o cuantificación de la calidad de vida. Evalúa la actitud frente al envejecimiento, la insatisfacción con la soledad y la ansiedad con un objetivo claro, el de medir el grado subjetivo de satisfacción de la persona anciana. Su aplicabilidad es excelente y sólo se ve limitada o interferida por la presencia de trastornos del lenguaje (afasias) y deterioro cognitivo.

Cuestionario "The Facts on Aging Quiz-1" (FAQ-1 de Palmore, 1998). Permite evaluar los conocimientos y sesgos actitudinales ante la vejez y el envejecimiento. Consta de 25 preguntas referentes a aspectos físicos, mentales y sociales así como diferentes estereotipos y los sujetos deben elegir la respuesta que ellos consideran correcta, mediante un formato de elección múltiple de 4 alternativas. Las puntuaciones totales pueden variar de 0 a 25 , de tal forma que la puntuación más alta refleja un mayor nivel de conocimiento sobre el envejecimiento y la salud de las personas mayores.

Cuestionario de atributos negativos y potencial positivo de la vejez (AN-PP; Morgan y Bengtson, 1976). Está integrada por las 14 afirmaciones originales ante las que expresar el grado de acuerdo/desacuerdo en escalas tipo Likert de cuatro puntos (totalmente en desacuerdo $=0$, ligeramente en desacuerdo $=1$, ligeramente de acuerdo $=2$, y totalmente de acuerdo $=3$ ). Ejemplos de ítems son: "Muchos ancianos pueden hacer un trabajo tan bien como las personas jóvenes pero no se les da la oportunidad para mostrar lo que pueden hacer" o "Muchos ancianos son inflexibles e incapaces de cambiar"

Cuestionario de datos sociodemográficos. Fue diseñado a medida para la presente investigación. Recogía datos tales como: edad, sexo, estado civil, convivencia y cuidado de personas mayores, situación laboral, opción política, creencias religiosas y práctica de la religión y conocimientos autopercibidos acerca de la vejez que los sujetos encuestados deberían contestar sobre sí mismos.

\section{Procedimiento}

Los cuestionarios fueron aplicados entre los meses de septiembre de 2014 y abril de 2015, utilizando para la recogida de datos el método de la bola de nieve. La población fue estratificada en múltiples etapas, el primer estrato fue la provincia, el segundo, el tamaño del municipio y, el tercero, el grupo etario.

A cada participante se le entregó un único documento que contenía los instrumentos de medida descritos en el apartado anterior.

Antes de entregar los cuestionarios, se les informó del carácter voluntario y anónimo de los mismos y les fue explicado el motivo de la encuesta y los objetivos que se pretendían alcanzar con la realización del estudio.

\section{Resultados}

Tal y como se puede observar en la Tabla 1, la edad media de las personas jóvenes fue de 25.76 años, la de las personas adultas, de 47.65, y la de las personas mayores de 65, de 74.56. De esta forma, la edad media de la totalidad de la población objeto de estudio, se situó en casi 50años.

Tabla 1.

Edad media de los participantes

\begin{tabular}{lcc}
\hline Grupos de edad & Media & Sx \\
\hline Edad 18-35 & 25.76 & 4.74 \\
Edad 36-64 & 47.65 & 7.15 \\
Edad $>65$ & 74.56 & 7.38 \\
Global & 49.68 & 21.05 \\
\hline
\end{tabular}

Como se puede observar en la tabla 2, en el grupo de participantes jóvenes, fueron halladas diferencias estadísticamente significativas en la subescala del AN-PP "Potencial positivo y características positivas en las personas mayores" $(\mathrm{F}=4.24 ; p=.042)$ en función de la práctica religiosa, reflejándose que son los jóvenes practicantes quienes presentaron niveles más elevados de actitudes positivas.

También fueron detectadas diferencias estadísticamente significativas en los niveles de conocimientos sobre el envejecimiento y sus procesos asociados (FAQ-1) entre las personas creyentes practicantes y no practicantes del grupo de jóvenes $(\mathrm{F}=9,14 ; p=.003)$, siendo las creyentes practicantes quienes muestran niveles más elevados de conocimientos.

Asimismo, fueron detectadas diferencias estadísticamente significativas entre jóvenes creyentes practicantes y no practicantes en el sesgo negativo y la tendencia actitudinal. El sesgo negativo, es más elevado en las personas religiosas no practicantes que en las practicantes y, la tendencia actitudinal, dentro de que es negativa en ambos casos, las personas jóvenes no practicantes tienen una tendencia actitudinal más negativa respecto a la vejez y sus procesos asociados que las practicantes. 
Tabla 2.

Conocimientos y actitudes hacia el envejecimiento en un grupo de jóvenes de 18 a 35 años en función de la práctica religiosa

\begin{tabular}{|c|c|c|c|c|}
\hline & $\begin{array}{c}\text { Religioso } \\
\text { practicante } \\
\mathrm{N}=16 \\
\text { Media }(\mathrm{Sx})\end{array}$ & $\begin{array}{c}\text { Religioso no } \\
\text { practicante } \\
\mathrm{N}=82 \\
\text { Media (Sx) }\end{array}$ & $\mathrm{F}$ & Sig. \\
\hline PGCMS & $\begin{array}{c}3.62 \\
(1.20) \\
\end{array}$ & $\begin{array}{c}3.49 \\
(1.29) \\
\end{array}$ & .140 & .709 \\
\hline AN-PP_Positivo & $\begin{array}{l}27.44 \\
(3.95) \\
\end{array}$ & $\begin{array}{l}25.36 \\
(3.63) \\
\end{array}$ & 4.240 & .042 \\
\hline AN-PP_Negativo & $\begin{array}{l}13.56 \\
(1.82) \\
\end{array}$ & $\begin{array}{l}13.34 \\
(1.91) \\
\end{array}$ & .182 & .670 \\
\hline AN-PP_Total & $\begin{array}{l}54.56 \\
(6.86) \\
\end{array}$ & $\begin{array}{l}52.05 \\
(6.01) \\
\end{array}$ & 2.235 & .138 \\
\hline FAQ-1 & $\begin{array}{l}11.50 \\
(2.92) \\
\end{array}$ & $\begin{array}{c}9.21 \\
(2.75) \\
\end{array}$ & 9.136 & .003 \\
\hline Sesgo positivo & $\begin{array}{c}3.69 \\
(1.08) \\
\end{array}$ & $\begin{array}{c}3.57 \\
(1.92) \\
\end{array}$ & .053 & .818 \\
\hline Sesgo negativo & $\begin{array}{c}8.06 \\
(2.91) \\
\end{array}$ & $\begin{array}{l}10.36 \\
(2.62) \\
\end{array}$ & 9.969 & .002 \\
\hline $\begin{array}{l}\text { Tendencia } \\
\text { actitudinal }\end{array}$ & $\begin{array}{l}-4.37 \\
(3.46)\end{array}$ & $\begin{array}{l}-6.79 \\
(3.70)\end{array}$ & 5.836 & .018 \\
\hline
\end{tabular}

En la tabla 3 se ponen de manifiesto los datos referentes a los conocimientos y actitudes hacia el envejecimiento en el grupo de adultos de 36-64 años en función de la práctica religiosa. En este caso, no encontramos ninguna diferencia estadísticamente significativa.

Tabla 3.

Conocimientos y actitudes hacia el envejecimiento en un grupo de adultos de 36-64 años en función de la práctica religiosa

\begin{tabular}{cccccc}
\hline & $\begin{array}{c}\text { Religioso } \\
\text { practicante } \\
\text { N=16 } \\
\text { Media (Sx) }\end{array}$ & $\begin{array}{c}\text { Religioso no } \\
\text { practicante } \\
\text { N=82 } \\
\text { Media (Sx) }\end{array}$ & F & Sig. \\
\hline PGCMS & $\begin{array}{c}2.31 \\
(1.59)\end{array}$ & $\begin{array}{c}2.68 \\
(1.57)\end{array}$ & 1.548 & .216 \\
\hline AN-PP_Positivo & $\begin{array}{c}26.20 \\
(3.15)\end{array}$ & $\begin{array}{c}25.29 \\
(3.73)\end{array}$ & 1.822 & .179 \\
\hline AN-PP_Negativo & $\begin{array}{c}12.46 \\
(1.93)\end{array}$ & $\begin{array}{c}12.38 \\
(2.32)\end{array}$ & .035 & .853 \\
\hline AN-PP_Total & $\begin{array}{c}51.13 \\
(5.33)\end{array}$ & $\begin{array}{c}50.05 \\
(6.81)\end{array}$ & .774 & .380 \\
\hline FAQ-1 & $\begin{array}{c}8.85 \\
(1.75)\end{array}$ & $\begin{array}{c}9.21 \\
(2.34)\end{array}$ & .774 & .381 \\
\hline Sesgo positivo & $\begin{array}{c}3.64 \\
(1.88)\end{array}$ & $\begin{array}{c}3.74 \\
(1.70)\end{array}$ & .096 & .757 \\
\hline Sesgo negativo & $\begin{array}{c}10.15 \\
(1.91)\end{array}$ & $\begin{array}{c}10.08 \\
(2.53)\end{array}$ & .023 & .879 \\
\hline Tendencia & $\begin{array}{c}-6.51 \\
\text { actitudinal }\end{array}$ & $\begin{array}{c}-6.34 \\
(3.28)\end{array}$ & .066 & .797 \\
\hline
\end{tabular}

Por lo que respecta al grupo de personas de mayor edad (ver tabla 4), fueron detectadas diferencias estadísticamente significativas en las puntuaciones de la subescala AN-PP "Potencial positivo y características personales positivas en las personas mayores" $(\mathrm{F}=4.63$; $p=033$ ), reflejándose que son los sujetos mayores practicantes quienes presentaron niveles más elevados de actitudes positivas.

Tabla 4.

Conocimientos y actitudes hacia el envejecimiento en un grupo de personas mayores de 65 años en función de la práctica religiosa

\begin{tabular}{cccccc}
\hline & $\begin{array}{c}\text { Religioso } \\
\text { practicante } \\
\text { N=16 } \\
\text { Media (Sx) }\end{array}$ & $\begin{array}{c}\text { Religioso no } \\
\text { practicante } \\
\text { N=82 } \\
\text { Media (Sx) }\end{array}$ & F & Sig. \\
\hline PGCMS & $\begin{array}{c}1.69 \\
(1.46)\end{array}$ & $\begin{array}{c}1.83 \\
(1.61)\end{array}$ & .249 & .619 \\
\hline AN-PP_Positivo & $\begin{array}{c}26.81 \\
(3.93)\end{array}$ & $\begin{array}{c}25.32 \\
(3.27)\end{array}$ & 4.628 & .033 \\
\hline AN-PP_Negativo & $\begin{array}{c}12.69 \\
(2.23)\end{array}$ & $\begin{array}{c}12.73 \\
(2.53)\end{array}$ & .008 & .927 \\
\hline AN-PP_Total & $\begin{array}{c}52.19 \\
(6.89)\end{array}$ & $\begin{array}{c}50.78 \\
(6.95)\end{array}$ & 1.227 & .270 \\
\hline FAQ-1 & 10.26 & $\begin{array}{c}10.49 \\
(2.39)\end{array}$ & .226 & .635 \\
\hline
\end{tabular}

Tabla 4

Conocimientos y actitudes hacia el envejecimiento en un grupo de personas mayores de 65 años en función de la práctica religiosa (continuación)

\begin{tabular}{ccccc}
\hline & $\begin{array}{c}\text { Religioso } \\
\text { practicante } \\
\text { N=16 } \\
\text { Media (Sx) }\end{array}$ & $\begin{array}{c}\text { Religioso no } \\
\text { practicante } \\
\text { N=82 } \\
\text { Media (Sx) }\end{array}$ & F & Sig. \\
\hline Sesgo positivo & $\begin{array}{c}3.40 \\
(1.79)\end{array}$ & $\begin{array}{c}3.58 \\
(1.56)\end{array}$ & .323 & .571 \\
\hline Sesgo negativo & 9.42 & $\begin{array}{c}9.02 \\
(2.79)\end{array}$ & .711 & .401 \\
\hline $\begin{array}{c}\text { Tendencia } \\
\text { actitudinal }\end{array}$ & $\begin{array}{c}-6.02 \\
(3.76)\end{array}$ & $\begin{array}{c}-5.44 \\
(2.77)\end{array}$ & .802 & .372 \\
\hline
\end{tabular}

Por otra parte, según se desprende de la tabla 5, existen diferencias estadísticamente significativas entre las personas jóvenes con diferentes orientaciones políticas en los resultados del cuestionario "Atributos negativos y potencial positivo de la vejez (AN-PP)". En concreto, estas diferencias se dieron en la subescala "Potencial positivo y características personales positivas en las personas mayores". Si profundizamos en el estudio, los análisis post hoc nos revelan que las diferencias se producen entre los jóvenes con orientaciones políticas de izquierdas y los de centro, siendo los primeros quienes presentaron niveles más elevados de actitudes positivas. 
Tabla 5.

Conocimientos y actitudes hacia el envejecimiento en un grupo de jóvenes de 18-35 años en función de la opción política

\begin{tabular}{|c|c|c|c|c|c|}
\hline & $\begin{array}{c}\text { Derechas } \\
\mathrm{N}=11 \\
\text { Media } \\
(\mathrm{Sx})\end{array}$ & $\begin{array}{c}\text { Centro } \\
\mathrm{N}=42 \\
\text { Media } \\
(\mathrm{Sx})\end{array}$ & $\begin{array}{c}\text { Izquierdas } \\
\mathrm{N}=68 \\
\text { Media } \\
(\mathrm{Sx})\end{array}$ & $\mathrm{F}$ & Sig. \\
\hline PGCMS & $\begin{array}{c}3.54 \\
(1.37)\end{array}$ & $\begin{array}{c}3.66 \\
(1.20)\end{array}$ & $\begin{array}{c}3.64 \\
(1.12)\end{array}$ & .041 & .960 \\
\hline AN-PP_Positivo & $\begin{array}{l}26.64 \\
(2.84) \\
\end{array}$ & $\begin{array}{l}23.98 \\
(3.57) \\
\end{array}$ & $\begin{array}{l}26.81 \\
(3.39) \\
\end{array}$ & 9.287 & .000 \\
\hline AN-PP_Negativo & $\begin{array}{l}13.45 \\
(1.37) \\
\end{array}$ & $\begin{array}{l}12.90 \\
(1.88) \\
\end{array}$ & $\begin{array}{l}13.47 \\
(1.95) \\
\end{array}$ & 1.226 & .297 \\
\hline FAQ-1 & $\begin{array}{l}10.00 \\
(3.03)\end{array}$ & $\begin{array}{c}9.57 \\
(2.79) \\
\end{array}$ & $\begin{array}{c}9.40 \\
(2.49) \\
\end{array}$ & .262 & .770 \\
\hline Sesgo positivo & $\begin{array}{c}3.54 \\
(1.63) \\
\end{array}$ & $\begin{array}{c}3.36 \\
(2.16) \\
\end{array}$ & $\begin{array}{c}3.53 \\
(1.48) \\
\end{array}$ & .135 & .874 \\
\hline Sesgo negativo & $\begin{array}{c}9.91 \\
(2.59) \\
\end{array}$ & $\begin{array}{l}10.28 \\
(2.30)\end{array}$ & $\begin{array}{l}10.22 \\
(2.89)\end{array}$ & .087 & .917 \\
\hline $\begin{array}{l}\text { Tendencia } \\
\text { actitudinal }\end{array}$ & $\begin{array}{l}-3.36 \\
(3.32)\end{array}$ & $\begin{array}{l}-6.93 \\
(3.58)\end{array}$ & $\begin{array}{l}-6.69 \\
(3.76)\end{array}$ & .119 & .887 \\
\hline
\end{tabular}

En la tabla 6 se exponen los datos relativos a los conocimientos y actitudes hacia el envejecimiento en el grupo de adultos de 36-64 años en opción política. Como se puede comprobar, no se detectaron diferencias estadísticamente significativas.

Tabla 6.

Conocimientos y actitudes hacia el envejecimiento en un grupo de adultos de 36-64 años en función de la opción política

\begin{tabular}{cccccc}
\hline & $\begin{array}{c}\text { Derechas } \\
\mathrm{N}=25 \\
\text { Media } \\
(\mathrm{Sx})\end{array}$ & $\begin{array}{c}\text { Centro } \\
\mathrm{N}=51 \\
\text { Media } \\
(\mathrm{Sx})\end{array}$ & $\begin{array}{c}\text { Izquierdas } \\
\mathrm{N}=56 \\
\text { Media } \\
(\mathrm{Sx})\end{array}$ & $\mathrm{F}$ & Sig. \\
\hline \multirow{2}{*}{ PGCMS } & $\begin{array}{c}2.28 \\
(1.93)\end{array}$ & $\begin{array}{c}2.68 \\
(1.48)\end{array}$ & $\begin{array}{c}2.95 \\
(1.51)\end{array}$ & 1.554 & .215 \\
\hline \multirow{2}{*}{ AN-PP_Positivo } & $\begin{array}{c}26.92 \\
(3.30)\end{array}$ & $\begin{array}{c}25.41 \\
(3.81)\end{array}$ & $\begin{array}{c}25.16 \\
(3.72)\end{array}$ & \multirow{2}{*}{2.070} & .130 \\
\hline \multirow{2}{*}{ AN-PP_Negativo } & $\begin{array}{c}12.12 \\
(2.26)\end{array}$ & $\begin{array}{c}12.61 \\
(2.22)\end{array}$ & $\begin{array}{c}12.39 \\
(2.24)\end{array}$ & \multirow{2}{*}{.409} & .665 \\
\hline \multirow{2}{*}{ FAQ-1 } & $\begin{array}{c}9.24 \\
(1.94)\end{array}$ & $\begin{array}{c}8.76 \\
(2.01)\end{array}$ & $\begin{array}{c}9.52 \\
(2.32)\end{array}$ & \multirow{2}{*}{1.671} & .192 \\
\hline \multirow{2}{*}{ Sesgo positivo } & $\begin{array}{c}3.84 \\
(1.70)\end{array}$ & $\begin{array}{c}3.69 \\
(1.65)\end{array}$ & $\begin{array}{c}3.64 \\
(1.63)\end{array}$ & \multirow{2}{*}{.124} & .883 \\
\hline \multirow{2}{*}{ Sesgo negativo } & 9.56 & 10.61 & 9.86 & \multirow{2}{*}{1.975} & .143 \\
& $(2.53)$ & $(2.30)$ & $(2.55)$ & \\
\hline Tendencia & -5.72 & -6.92 & $-6-21$ & \multirow{2}{*}{1.091} & .339 \\
actitudinal & $(3.58)$ & $(3.50)$ & $(3.57)$ & & \\
\hline
\end{tabular}

Finalmente, en relación al grupo de personas mayores, tal y como queda reflejado en la tabla 7 , fueron halladas diferencias estadísticamente significativas en función de la opción política en el cuestionario de "Atributos negativos y potencial positivo de la vejez (AN-PP)". Estas diferencias se dieron una vez más en la subescala "Potencial positivo y características personales positivas en las personas mayores". Los análisis a posteriori revelan que tales diferencias se concentraban entre los participantes de centro y los de derechas, reflejándose que son las personas mayores con orientación política de derechas las que presentaron niveles más elevados de actitudes positivas.

Tabla 7.

Conocimientos y actitudes hacia el envejecimiento en un grupo de personas mayores de 65 años en función de la opción política

\begin{tabular}{|c|c|c|c|c|c|}
\hline & $\begin{array}{c}\text { Derechas } \\
\mathrm{N}=39 \\
\text { Media } \\
(\mathrm{Sx})\end{array}$ & $\begin{array}{c}\text { Centro } \\
\mathrm{N}=44 \\
\text { Media } \\
(\mathrm{Sx}) \\
\end{array}$ & $\begin{array}{c}\text { Izquierdas } \\
\mathrm{N}=30 \\
\text { Media } \\
(\mathrm{Sx})\end{array}$ & $\mathrm{F}$ & Sig. \\
\hline PGCMS & $\begin{array}{c}1.87 \\
(1.47) \\
\end{array}$ & $\begin{array}{c}1.93 \\
(1.57) \\
\end{array}$ & $\begin{array}{c}1.52 \\
(1.64) \\
\end{array}$ & .660 & .519 \\
\hline AN-PP_Positivo & $\begin{array}{l}27.33 \\
(4.17) \\
\end{array}$ & $\begin{array}{l}25.02 \\
(2.86) \\
\end{array}$ & $\begin{array}{l}25.50 \\
(3.30) \\
\end{array}$ & 4.905 & .009 \\
\hline AN-PP_Negativo & $\begin{array}{l}12.38 \\
(2.38) \\
\end{array}$ & $\begin{array}{l}12.77 \\
(2.47) \\
\end{array}$ & $\begin{array}{l}12.93 \\
(2.36) \\
\end{array}$ & .491 & .613 \\
\hline FAQ-1 & $\begin{array}{l}10.10 \\
(2.76) \\
\end{array}$ & $\begin{array}{c}9.91 \\
(2.54) \\
\end{array}$ & $\begin{array}{l}10.13 \\
(2.50) \\
\end{array}$ & .086 & .918 \\
\hline Sesgo positivo & $\begin{array}{c}3.46 \\
(1.82) \\
\end{array}$ & $\begin{array}{c}3.68 \\
(1.93) \\
\end{array}$ & $\begin{array}{c}3.63 \\
(1.42) \\
\end{array}$ & .171 & .843 \\
\hline Sesgo negativo & $\begin{array}{c}9.31 \\
(2.87) \\
\end{array}$ & $\begin{array}{c}9.52 \\
(2.66) \\
\end{array}$ & $\begin{array}{c}9.37 \\
(2.31) \\
\end{array}$ & .073 & .930 \\
\hline $\begin{array}{l}\text { Tendencia } \\
\text { actitudinal }\end{array}$ & $\begin{array}{l}-5.85 \\
(3.75) \\
\end{array}$ & $\begin{array}{l}-5.84 \\
(3.87) \\
\end{array}$ & $\begin{array}{l}-5.73 \\
(3.04) \\
\end{array}$ & .010 & .990 \\
\hline
\end{tabular}

\section{Discusión}

Por lo que respecta a la práctica religiosa, no hemos encontrado estudios en la literatura científica de nuestro entorno relacionados con este tema. La mayoría de la población gallega es de religión católica (IGE, 2015). Los datos de nuestro estudio revelan que: el $36,6 \%$ de los jóvenes encuestados son creyentes $y$, de ese porcentaje, un $16,3 \%$ son practicantes; el $68,2 \%$ del grupo de personas adultas encuestadas son creyentes aunque únicamente el $29,3 \%$ de las mismas son practicantes. Por último, en lo que atañe al grupo de adultos mayores de 65 años, cabe destacar que el 95, 2\% son creyentes, manifestándose como practicantes de la religión católica un $71,7 \%$ de los mismos. En este sentido, cabe destacar que, en general, las personas que respondieron que eran practicantes, tenían estereotipos explícitos más positivos hacia la vejez y sus procesos asociados en el grupo de jóvenes y de personas mayores.

En línea con la variable analizada con anterioridad, cuando hablamos de la opción política, tampoco encontramos estudios, en la literatura científica de nuestro entorno, relacionados con este tema. Nuestra investigación pone de manifiesto que se dan actitudes significativamente más positivas frente a la vejez y sus procesos asociados en el grupo de jóvenes con orientaciones políticas de izquierda con respecto a los de centro. También, se dan actitudes significativamente más positivas frente a la vejez y sus procesos asociados en el grupo de personas mayores con orientaciones políticas de derechas con respecto al de centro. 


\section{Referencias}

Gaviria, E., López, M. \& Cuadrado, I. (2013). Introducción a la psicología social ( $2^{\mathrm{a}}$ Ed.). Madrid: Sanz y Torres.

Eagly, A. \& Chaiken, S. (2005). Attitude research in the 21st century: The current state of knowledge. En D. Albarracin, B. Jonson \& M. Zanna (Eds.), The Hanbook of attitudes (pp. 743-767). Mahwah, New Jersey: Lawrence Erlbaum.

IGE (2015). Principais datos de Galicia. Recuperado de http://www.ige.eu/igebdt/esq.jsp?pagina $=002005 \&$ rut $\mathrm{a}=$ datos-basicos/datos_basicos.jsp\&idioma $=\mathrm{ga}$

Lawton, M.P. (1975). The Philadelphia Center Morale Scale: a revisión. Journal of Marriage and the Family, 56, 559-565. http://dx.doi.org/10.2307/352867

Morgan, L.A. \& Bengtson, V.L. (1976). Negative attributes of old age and positive potential in old age. En D.J. Magen \& W.A. Peterson (Eds.), Research instruments in social gerontology (pp.606-607). Minneapolis, MN: University of Minnesota Press.

Palmore, E.B. (1998). The Facts on Aging Quiz ( $2^{\mathrm{a}}$ Ed.). Nueva York: Springer.

Villar, F. \& Triadó, C. (2000). Conocimiento sobre el envejecimiento: Adaptación del FAQ (Facts on Aging Quiz) y evaluación en diferentes cohortes. Revista de Psicología General y Aplicada, 53(3), 523-534. http://www.ub.edu/dppsed/fvillar/principal/pdf/2000a _faq.pdf 